André Burger,

Genève

\title{
SUR UN DEPLACEMENT DE VALEURS: TRAIRE ET TIRER
}

L'étymologie de tirer est inconnue. Celle que le regretté W. von Wartburg a cru. pouvoir avancer est inacceptable: tirer serait sorti de martyrier par la grâce d'une fausse coupure mar tirier. Il est plus qu'improbable que cette «étymologie populaire» eût pu se produire si la langue ne possédait pas au préalable un v'erbe tirier. Au surplus aucun fait n'appuie cette hypothèse. Le $F E W, \mathrm{t}$. VI, 1, p. 396, donne bien pour tirer le sens de «torturer sur un tréteau», au XIIIe siècle, et p. 403, «démembrer en faisant tirer les quatre membres par des chevaux» et «torturer (qn) en l'étendant sur un tréteau» au XIVe siècle; ces sens sont évidemment trop tardifs pour permettre des conclusions sur l'origine de tirer, d'autant plus qu'ils s'expliquent sans peine par la valeur normale du mot, attesté dès la Chanson de Roland, sans aucun rapport avec martirie, mot savant de clerc, qui, dans le même texte s'applique deux fois sur quatre exemples aux Sarrasins, vv. 501 et 1467 , où il ne signifie pas «martyre» mais «massacre»; martirier n'apparaît qu’à partîr de Wace (FEW VI, 1, p. 397). Il faut dès lors aussi récuser l'idée de Wartburg, fondée sur son étymologie et non sur les textes, que tirer aurait à l'origine »einen starken affektiven unterton« ( $F E W$ XIII, 2, p. 185). L'étude des exemples de la Chanson de Roland, confrontés avec ceux de traire, nous amènera à une conclusion toute différente.

On relève dans la Chanson de Roland 14 exemples de traire comportant les acceptions suivantes:

10 «tirer (l'épée du fourreau)»:

1367 Danz Oliver trait ad sa bone espee

et de même 811 (seient... traites), 1324 (trait), 1365 (traire), 2089 (il trait), 3431 (trait ad), 3402 et 3576 (ont traites), soit plus de la moitié des exemples.

$2^{0}$ «tirer (une flèche)»:

2265 Plus qu' arcbaleste ne poet traire un quarrel

(Dun a. $O$; corr. d'après $V_{4} P T L$ ) 
30 «tirer (ses cheveux, sa barbe)»:

2596 Trait ses chevels, si se cleimet caitive

et de même 2906 (trait ses crignels), à quoi on peut ajouter le composé detraire:

2930 Sa barbe blanche cumencet a detraire,

Ad ambes mains les chevels de sa teste.

$4^{0}$ «amener (quelqu'un)»:

$3749 \mathrm{Li}$ emperere devant sei l'ad fait traire.

et au réfléchi:

2131 Dist l'un a l'altre: Ça vus traiez ami.

$5^{0}$ «se rapprocher de, ressembler à»:

3177 Granz est e forz e trait as anceisors

En face des 14 exemples et de la polysémie de traire, tirer n'apparaît que 5 fois et toujours au sens de «exercer un effort de traction sur un objet, sans le déplacer notablement», soit le sens $3^{\circ}$ de traire:

2414. Tire sa barbe, cum hom qui est iret

et de même 2943, 3712, 4001,

Au vers 2283, il s'agit de l'épée que Roland évanoui tient dans sa main et dont un Sarrasin tente de s'emparer:

2280 Rollant saisit e son cors e ses armes

E dist un mot: «Vencut est li nies Carles!

Iceste espee porterai en Arabe.»

En cel tirer li quens s'aperçut alques.

(tireres $O$ )

Co sent Rollant que s'espee li tolt

Il ne s'agit pas ici de tirer l'épée du fourreau; le Sarrasin tente d'arracher l'épée de la main de Roland, mais sans y réussir: au vers 2284 on a $l i$ tolt et non $l i$ ad tolut; Bédier traduit correctement en cel tirer par «comme il tirait»; il s'agit donc, comme dans les autres exemples d'un effort de traction qui ne déplace pas notablement l'objet.

A s'en tenir au texte de la Chanson de Roland, la relation entre traire et tirer est parfaitement claire: il s'agit d'une relation d'inclusion; traire est le terme dont l'extension est la plus grande, le terme incluant; tirer ne recouvre qu'une des acceptions de traire, il est le terme inclus. C'est le type de relation qu'on a, par exemple, en latin, entre homo et uir. On peut l'illustrer graphiquement par la figure:

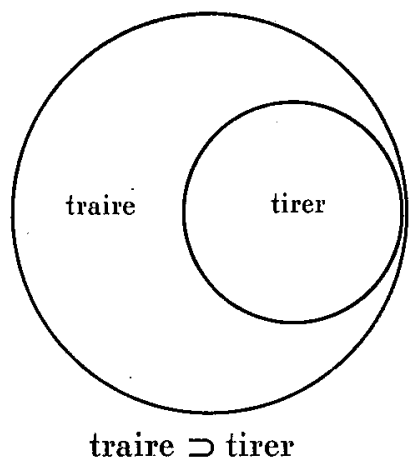


On pourrait objecter que cette conclusion repose sur un nombre trop restreint d'exemples pour être assurée; mais elle le deviendra en observant que la relation entre nos deux termes est exactement la même chez Chrétien de Troies qui passe pour le meilleur représentant de la langue de son temps (aux alentours de 1170).

Le dictionnaire de Foerster, qui n'est pas exaustif mais donne soigneusement les diverses acceptions des mots, fournit 73 références pour traire et 11 pour tirer. Pour le premier, la gamme des acceptions est un peu plus riche que dans la Chanson de Roland, mais la valeur est la même. On retrouve le sens «tirer' (l'épée)» p. ex. Erec 3836 (Roques):

Sore lui cort, espee traite

celui de «tirer (une flèche)», Graal 7231:

Ne arbaleste n'i traisist

de «tirer (ses cheveux, etc.)», Erec 3790 (Roques):

Ses poinz tordre, ses chevox traire

de «faire venir», Lion 2547:

La dame en a consoil treite

au réfléchi «aller», Graal 182

Maintenant pres de lui s'est trait

On ne retrouve pas le sens de «ressembler»; en revanche on trouve parole traire (de quelqu'un) «en tirer un mot», Graal 8072; la paine qu'elle aboit traite «endurée», Graal 4056; si en trai a garant Macrobe, Erec 6676 (Roques) «j'en prends pour caution M.», qui tous s'expliquent sans peine en partant du sens général de «tirer».

Quant à tirer, le sens en est le même que dans le Roland, mais avec des contextes un peu plus variés. On trouve naturellement le sens de «tirer (ses cheveux)», Lion 1159:

Ses chevos tire et ront ses dras

et de même Erec 4305 (Roques), Graal 8461; en outre un exemple comparable à celui de Rol. 2283; Perceval cherche à s'emparer de l'épée du Chevalier Vermeil qu'il vient de tuer, mais n'y parvient pas; Graal 1126 ss.:

Et s'a talent qu'il li desçaigne

l'espee, mais il nel set faire,

ne del fuerre ne le puet traire

ains prent l'espee et sache et tire

et il faut qu'Yvonnet lui montre comment s'y prendre.

Puis on trouve quatre exemples au sens de «tirer (quelqu'un par son vêtement, etc., pour attirer son attention)»; Graal 258:

Et li valles le tenoit pris

Par le haubert et si le tire

pour lui demander à quoi sert ce haubert; de même 4769, 5343, 7663. 
Il est enfin employé dans une description de lutte à main plate entre Erec et un chevalier; Erec 5953 (Roques):

Erec a son talent le mene et sache et tire

et finit par le faire tomber.

Ces deux dernières acceptions découlent aisément de la valeur de tirer: «exercer un effort de traction sur un objet, sans déplacement notable». Mais on a là peut-être l'amorce de l'extension du sens de tirer; dans un exemple comme Graal 7663:

Et li notonniers a lui tire

Monseignor Gavain et dist:

on peut concevoir que le nautonier prenne à part Gauvain en le faisant se déplacer; et dans Erec 5953, l'effort d'Erec finit par faire tomber son adversaire.

Nous ne pouvons pas suivre ici le détail de l'évolution de nos deux termes et nous nous bornerons à renvoyer au $F E W$, t. XIII, 2, p. 177 ss. pour traire et t. VI, 1, p. 397 ss. pour tirer. Il en ressort qu'entre le XIIe siècle et le XVe, tirer a peu à peu occupé tontes les positions de traire. Villon n'emploie pas traire, mais il a trois exemples de tirer: T 1333 qu'on leur tire les oreilles; 1546 tire toy pres; 1872 que l'on tire mon estature «que l'on trace mon portrait».

Au siècle suivant, je relève dans le Gargantua (éd. Lefranc) 21 exemples de tirer contre deux de traire; de ces derniers, l'un au sens moderne: VII, 20 elle poubait traire de ses mammelles quatorze cens deux pipes neuf potees de laict; l'autre au sens de «tracer (des caractères): XXIII, 97 bien traire et former les antiques et romaines lettres.

Pour tirer, on trouve: XLVII, 58 ss. Touquedillon [...] tyra son espee et en transperça Hastiveau [...] Et, tyrant son coup du corps, dist; XI, 31 tiroit au cheorotin; XXIII, 163 affeustait le canon, tyroit à la butte; XXXVI, 26 luy tyra un coup de canon; 34 luy tirerent plus de neuf mille vingt et cinq coups de faulconneaux et arquebouzes; 36 si menu tiroient contre luy; 41 les coups d'artillerie que l'on tiroit du chasteau; XI, 33 tiroyt d'un sac deux moustures; XII, 72 Alors [...] qu'on feist de vostre nez une dille pour tirer un muy de merde; XXXV, 7 Bon Joan [...] tyra ses heures de sa braguette; XV, 46 et ne fut possible de tirer de luy une parole; XL, 22 il ne tire pas l'aroy comme le boeuf; XXIII, 142 tyrant par les dens son manteau (en nageant); XXXI, 65 si l'esperit calumniateur tentant a mal te tirer; XL, 13 le froc et la cogule tire a soy les opprobres; XXXV, 64 tyrant droict son chemin oer's La Vauguyon; XLVIII, 49 Le moyne tira pers le fort et tant feist qu'il monta sus; XLII, 24 tyrant vers La Saullsaye; XXXIII, 20 L'aultre partie, cependent, tirera vers Onys; XXIV, 14 comment on tiroit les métaux; XLI, 35 les faulconniers, davant que paistre leurs oyseaux, les font tyrer quelque pied de poulle; II, 81. selon la loy que l'on tire au rivet; LI, 9. tirer les presses a son imprimerie. 
Le contraste est éclatant et si on relève encore au XVIe siècle un bon nombre d'exemples de traire (25 dans le Dictionnaire de Huguet), ils font l'impression d'archaïsmes littéraires ou d'emploi technique (or trait «fil d'or»). Toutefois tout lien sémantique n'est pas rompu entre traire et tirer: on peut non seulement substituer tirer à traire dans l'expression traire du lait, mais on trouve encore l'exemple suivant d'Amyot, cité par Godefroy, t. VII, p. 728 les tirouers ou elle tiroit ses brebis «les seanx à traire où elle trayait ses brebis». Cela signifie que la relation entre traire et tirer s'est complètement retournée; c'est maintenant tirer qui est le terme général et qui inclut traire, terme spécial, soit:

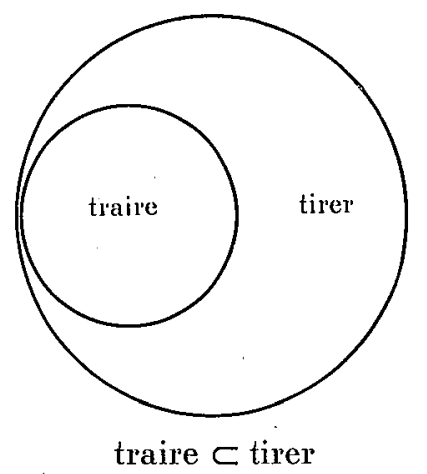

Mais avec le triomphe, au XVIIe siècle, du purisme, qui réprouve l'archaïsme, traire ne subsiste plus qu'au sens moderne. Le Dictionnaire de l'Académie (1696) ne consacre à traire que cette brève mention: «Traire v. a. Tirer. Il n'a guere d'usage qu'en cette phrase: traire les vaches, pour dire tirer le lait du pis des vaches». Le lien sémantique est. rompu; entre traire et tirer on n'a plus qu'une relation d'exclusion:
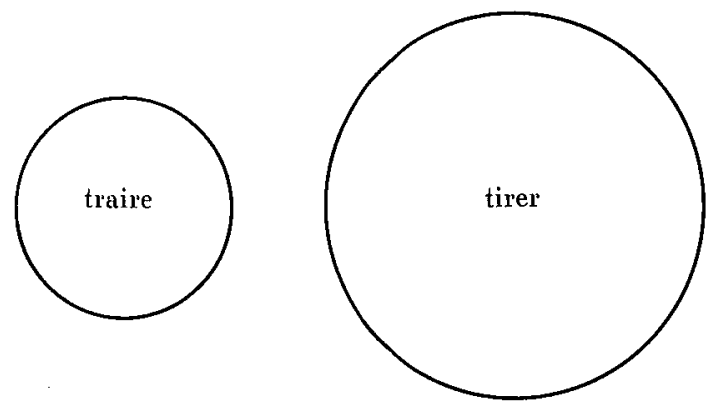

traire : tirer 
Povzetek

SEMANTICNI PREMIK VREDNOSTI GLAGOLOV TRAIRE IN TIRER

Avtor ne sprejema Wartburgove etimologije za tirer $(<\gg$ martyrier $\ll)$; ta glagol se pojavlja prepozno, da bi tako razlago lahko sprejeli.

Teksti namreč kažejo, da je $\mathrm{v}$ najstarejši dobi (Chanson de Roland, Chrétien de Troies) traire glagol, ki prekriva vse semantično polje, tirer pa samo del pomena. V poznejših stoletjih pa začne tirer prevladovati; že pri Villonu, še bolj pri Rabelaisu: v XVI. stol. je traire uporabljen ali močno literarno ali pa v docela tehničnem jeziku (in kot tak se je obdržal do naših dni). Situacija se je torej docela obrnila: zdaj je tirer tisti, ki prekriva celo semantično polje, traire pa samo ozek sektor ('molzti kravo'). 